\title{
"humanidades
}

Revista Humanidades

ISSN: 2215-3934

humanidades@ucr.ac.cr

Universidad de Costa Rica

Costa Rica

\section{El cuerpo como frontera biopolítica del reacondicionamiento sexual: más allá de lo biológico y semántico}

Sánchez- Valdez, Dr.(c) Ángel; Rico Bovio, Dr. Arturo

El cuerpo como frontera biopolítica del reacondicionamiento sexual: más allá de lo biológico y semántico

Revista Humanidades, vol. 11, núm. 1, 2021

Universidad de Costa Rica, Costa Rica

Disponible en: http://www.redalyc.org/articulo.oa?id=498064330006

DOl: https://doi.org/10.15517/h.v11i1.44151

\section{(c) (1) $\Theta($}

Esta obra está bajo una Licencia Creative Commons Atribución-NoComercial-SinDerivar 3.0 Internacional. 
Desde los estudios étnicos, culturales, de género y ambientales

\section{El cuerpo como frontera biopolítica del reacondicionamiento sexual: más allá de lo biológico y semántico}

The Body as a Biopolitical Frontier of Sexual Reconditioning: Beyond the Biological and Semantic

Dr. (c) Angel Sánchez-Valdez

Universidad Autónoma de Chibuahua, México

msvaldez@uach.mx

(iD http://orcid.org/0000-0002-6927-294X

Dr. Arturo Rico Bovio

Universidad Autónoma de Chibuahua, México

arico@uach.mx

(DD http://orcid.org/0000-0002-6840-9625
DOI: https://doi.org/10.15517/h.v11i1.44151

Redalyc: http://www.redalyc.org/articulo.oa? id $=498064330006$

Recepción: 19 Mayo 2020

Aprobación: 21 Julio 2020

\section{Resumen:}

La presente investigación pretende identificar, a través de un análisis biopolítico de la corporalidad alternativa, los mecanismos y pautas institucionales empleadas por el Estado para la administración de la sexualidad y su diversidad. Este estudio integra, además, elementos biológico-genéticos como un andamiaje a considerar en el análisis de los alcances de la cirugía de reasignación de sexo para las personas transexuales, facilitada en el caso concreto del Estado español. Se encuentra que, en los últimos años, dentro del sistema capitalista, la organización y visibilización de la diversidad sexual a la cabeza de su colectivo (LGBTTTIQ+) ha ido estableciendo modificaciones a las políticas estatales respecto de la administración de la diversidad sexual, transformando modelos de persecución y victimización en pautas de inclusión y cuidado institucional.

Palabras clave: sexo, diversidad, género, sexualidad, órganos.

\section{Abstract:}

The present investigation tries to identify the mechanisms and institutional guidelines used by the State for the administration of sexuality and its diversity, through a biopolitical analysis of said alternative corporality. This study also integrating biologicalgenetic elements as a scaffolding to consider in the analysis of the scope of sex reassignment surgery for transsexual people facilitated in the specific case of the Spanish State. Finding that within the capitalist system in recent years, the organization and visibility of sexual diversity at the head of its collective (LGBTTTIQ +), has been establishing modifications to state policies regarding the administration of sexual diversity, transforming from persecution and victimization models in guidelines of inclusion and institutional care.

KEYWORDS: sex, diversity, gender, sexuality, organs.

\section{Comentarios PRELiminares SOBRE CUERPo Y SEXo}

Desde el establecimiento de la dicotomía clásica que fragmentó al ser humano en materia y espíritu, el cuerpo ha sido considerado morada temporal de lo inmaterial. Los sistemas socioeconómicos se han valido de discursividades, tanto teológicas como ético-morales, que lo han disciplinado, castigado y administrado. En contraste, estos discursos también han permitido el desarrollo de una conciencia sobre sí mismo, un cuestionamiento inagotable de sus alcances y potencialidades. Actualmente, la identidad sexual es considerada producto de un andamiaje biológico preestablecido (genético), así como también resultado de una construcción de roles y dinámicas sociales intrapersonales e interpersonales. 
La expresión de la sexualidad a través del cuerpo genera discursos diversos que manejan y vinculan diferentes componentes afectivos, biológicos y socioculturales, los cuales, usualmente, se adecúan a los parámetros definidos por la estructura social o el sistema político-económico dominante.

La relación entre simbología, representación y administración del cuerpo, de manera particular en el ámbito temático de la sexualidad, presenta retos complejos para su análisis porque involucra tratamientos y consideraciones médico-científicas, filosóficas y ético-morales. Esta complejidad exige, en nuestra opinión, proponer una metodología transdisciplinaria para su abordaje a fin de establecer referentes diversos que concurran al análisis, adoptando distintas herramientas y visiones históricas, científicas y filosóficas. El objetivo es alcanzar una comprensión más profunda de este intrincado fenómeno sociocultural que ha generado un amplio debate de escala mundial, a partir de la diferenciación entre sexo, género e identidades sexuales.

La dinámica del reacondicionamiento sexual "físico-psicológico" de las personas transexuales ${ }^{1}$ es un proceso complejo de larga duración en el cual se pretende compaginar, de la manera más armoniosa posible, los múltiples elementos de lo subjetivo (autopercepción y aceptación) y lo físico (caracteres sexuales secundarios) de la corporalidad, rediseñada en el caso concreto de los sujetos transexuales o simplemente trans.

A manera de aproximación metodológica se pretende, en primer lugar, mediante un análisis conjunto de pautas de la biopolítica y de la filosofía del cuerpo, identificar cuáles y de qué forma han operado algunas dinámicas y dispositivos institucionales del Estado encargados de administrar la expresión sexual de la corporalidad y su diversidad. En segundo término, se mencionan ciertos elementos contenidos en el Manual Diagnóstico de Trastornos Mentales publicado por la Asociación Americana de Psiquiatría, con el fin de identificar ciertas características y especificidades relacionadas a la transexualidad y la cirugía de reasignación de sexo ${ }^{2}$. En este sentido, consideramos pertinente la revisión de este manual para el análisis transdisciplinario sobre los efectos y resultados de la cirugía de reasignación de sexo, puesto que esta requiere de un equilibrado e integral acompañamiento en la medida que incluye ajustes y modificaciones tanto de elementos hormonales (testosterona, progesterona, estrógenos, etc.) como psicológicos (experiencia de vida, roles y dinámicas) y físico-corporales (características sexuales secundarias).

Finalmente y derivado del análisis de dichas condiciones, nos proponemos perfilar planteamientos que contemplen tanto factores biológicos como socioculturales, así como posibles pautas de aproximación transdisciplinaria que permitan realizar un abordaje integral de lo complejo y multifactorial de dicho proceso de resignificación de la corporalidad de la diversidad sexual.

\section{LAS NOCIONES DEL CUERPO}

Entre los conceptos clave para el abordaje de las diferentes estructuras y andamiajes del poder como ente dinámico que se desarrolla y ejerce influencia directa en los individuos están los referidos por Foucault, uno de ellos es el de tecnologías de disciplina, lo cual refiere a herramientas que han sido eficaces en la administración de la vida y del cuerpo del individuo y su colectividad, regulando acciones e imaginarios; pero, más allá de individuos en lo singular, en cuanto a colectivos individuales Foucault (1977) señala que:

El decir que el poder tomó posesión de la vida en el siglo diecinueve, o decir que el poder por lo menos tomó la vida bajo su cuidado [...] tuvo de manera particular éxito en cubrir toda la superficie que yace entre lo orgánico y lo biológico, entre el cuerpo y la población (p. 30).

Las tecnologías de disciplina eran pues todas aquellas medidas que el Estado adoptaba e implementaba para administrar la corporalidad, tanto individual como colectiva, con base en elementos propios de la moral sustentados, por un lado, por la estructura eclesiástica y de la evidencia científica respecto de una taxonomía de la normalidad por el otro. 
El cuerpo humano ha sido objeto de múltiples interpretaciones a lo largo de la historia, entre las cuales se encuentra algunas conceptualizaciones religiosas, principalmente occidentales, que constituyeron la dicotomía ontológica del cuerpo entre sus elementos psíquico y material, respectivamente, relación conocida como dualismo. Desde la antigüedad se desarrollaron a la par diversas dinámicas político-religiosas para la administración y control de los cuerpos (tributos, castigos, sacrificios, ayuno, penitencias, entre otros), con la confrontación entre monistas y dualistas que polemizaban sobre la naturaleza e importancia del cuerpo y el alma, pasando por una reinterpretación de la concepción de la naturaleza humana sostenida por el judeocristianismo que fue modificándose a lo largo de la Edad Media y la Modernidad.

En la edad moderna, la reforma religiosa y la secularización que de ella provino facilitaron el despegue de la investigación científica y el desarrollo del modelo capitalista de producción. Con este impulso se hizo factible que el positivismo y el historicismo florecieran junto con la Revolución Industrial, estableciendo las bases para la propuesta posmoderna que sostiene la tesis de la construcción y deconstrucción de la subjetividad humana que caracteriza los tiempos que vivimos.

En este devenir, la conceptualización del cuerpo ha sido objeto de innumerables definiciones, transformaciones, reivindicaciones y demás mecanismos que han buscado comprenderlo, ya sea para intentar liberarlo o, de alguna forma (pragmático-materialista), encontrar nuevas maneras para optimizar su administración y control según las leyes del sistema político-económico vigente. Le Breton recurre a la consideración del cuerpo como entidad simbólica, a diferencia del tradicional enfoque occidental positivista del cuerpo-ente sujeto de estudio, medición y escrutinio, en cuanto a su dimensión anatomo-fisiológica.

Entender el cuerpo como símbolo, a diferencia de lo meramente descriptivo, permite redimensionar y potencializar una multiplicidad de significados. Al respecto Le Breton (2002) señala que: "El cuerpo es una construcción simbólica, no una realidad en sí mismo. De ahí la miríada de representaciones que buscan darle un sentido y su carácter heteróclito, insólito, contradictorio de una sociedad a otra" (pp. 13-14). El símbolo lucha contra la absolutización hegemónica del cuerpo científizado para dar paso a interpretaciones que se apegan a contextos y latitudes específicas. Por nuestra parte postulamos que la corporeidad está constituida por diversos factores visibles e invisibles que funcionan como un solo sistema, aunque a menudo se interpretan, modifican y actúan por separado (taxonomía), por ende, suele dejarse de lado al todo corporal, el cual se torna en una realidad inaccesible y al mismo tiempo vulnerable.

El cuerpo recibe así múltiples etiquetas que no pretenden comprenderlo y explicarlo, sino que lo definen únicamente para controlarlo. Ante tal situación nuevas categorías se vuelven necesarias para buscar desentrañarle, al respecto Rico Bovio (2017) menciona: "Con esa intención he sugerido el término valencias corporales, para referirnos a las propiedades que un cuerpo tiene para relacionarse con otros cuerpos, semejantes o diferentes y consigo mismo" (p. 65). Dichas valencias permiten concebir las múltiples dimensiones que involucran la corporalidad, ya sean químicas, físicas, biológicas, psicológicas o sociales.

La interpretación histórico-social mudable del cuerpo humano lo ha vuelto indefenso y proclive a todo tipo de violencia, tanto discursiva como física, haciéndolo pasar por varias fases históricas de control, desde la monárquico-religiosa a la político-estatal moderna que persigue la explotación económica de los cuerpos. En los procesos de formación de los individuos dejan de tener importancia los conocimientos, puesto que las leyes y reglamentaciones tradicionales sufrirán a diferentes ritmos una sustitución derivada del biopoder. La función de la legalidad se modifica de modo tal que, en vez de funcionar como réferi que regula derechos inalienables, da un paso atrás en su función natural de favorecer la integración social, limitando su acción a representar intereses de carácter negociable, mientras neutraliza y silencia a los demás.

En cuanto a la fuerza y su relación con el campo político Foucault señala que:

Toda relación de fuerza implica en todo momento una relación de poder (que es en cierto modo su forma momentánea) y cada relación de poder reenvía, como a su efecto, pero también como a su condición de posibilidad, a un campo político del que forma parte. Decir que «todo es político» quiere decir esta omnipresencia de las relaciones de fuerza y su inmanencia en 
un campo político; pero además es plantearse la tarea hasta ahora esbozada de desembrollar esta madeja indefinida (Foucault, 1979 , p. 159).

La administración de la corporeidad no puede materializar sus postulados sin las dinámicas que condicionan dicha corporalidad y su expresión sexual; los mecanismos que utiliza ejercen influencia no solo en sus definiciones, interacciones y movilidad, sino en la percepción que tiene el colectivo (LGBTTTIQ+) y la sociedad en general sobre dicha expresión. El cuerpo como enigma sujeto a descubrimiento y, por tanto, al control, encuentra en la subjetividad un referente epistemológico inagotable.

El conocimiento sobre el cuerpo ha sido motivo de cuestionamiento crónico, poder, violencia y equilibrio, todos principios rectores en la búsqueda de su comprensión, por ello su definición es pues elusiva, como lo son también sus límites. La narrativa mitológica griega da cuenta de que Prometeo fue severamente castigado a vivir encadenado a una roca y a ser picoteado en sus entrañas en un tormento sin fin por haber llevado el "fuego" del conocimiento a los hombres. Esta alegoría pone de manifiesto el lugar privilegiado que ostenta el conocimiento para el ser humano y que no puede separarse de su correspondiente sufrimiento, pareciendo repetirse así el temor bíblico (testamentario) de estar transgrediendo los límites de un privilegio reservado a Dios. Desde la satisfacción de las necesidades o pulsiones básicas (o de supervivencia) en los tiempos de los primeros homínidos racionales, hasta las más intrincadas configuraciones teóricas sobre nuestra trascendencia como especie en el siglo XXI, el “castigo" por el conocimiento biológico-discursivo del cuerpo pudiese ser lo indeterminable e inacabado y, por tal motivo, lo sistemáticamente excluido.

La simbología que el cuerpo asume es múltiple y variada, encontrándose supeditada discursiva y metodológicamente tanto al contexto y a la época en que se vive como a las creencias y preferencias de quienes la generan. El común denominador es el cuerpo mismo, a manera de hilo conductor y referente de un largo proceso de reencuentro y reivindicación. Le Breton define al cuerpo como el medio por el cual es posible vincularse con el mundo, no solo como un dispositivo mecánico-sensorial, sino como algo que puede ser interminablemente interpretado. Con respecto a lo anterior el sociólogo señala que: "Vivir consiste en reducir continuamente el mundo al cuerpo, a través de lo simbólico que este encarna" (Le Breton, 2002, p. 7). Toda simbología corporal, en su propia dimensión, su tiempo y espacio, asumiría una definición que le permite estar en condiciones de aportar una medida que posibilita el análisis, explicación y comprensión de un presente.

La consciencia de la existencia corporal y sus posibles interpretaciones permiten la vinculación en un nivel axiológico de los componentes de la esencia humana, tanto natural como divina. La experiencia sensible del mundo se vuelve la base sobre la cual se construye una más amplia explicación de la realidad, configurándose como un dispositivo que a medida que es aplicado experimenta un proceso de especialización a partir de un esquema de ensayo-error.

Los sentidos se vuelven los medios que no solo posibilitan el contacto del hombre (como especie) con el mundo, sino que permiten la interpretación de dicha interacción; las variables, intensidades y predicciones de lo posible se vuelven parte fundamental del conocimiento que, a la postre, adquirirá una terminología que intentará definirlo y acondicionarlo desde un punto de vista científico, con repercusiones en lo social, humanístico, informático, religioso, administrativo, biológico, etc., fragmentaciones que responden a diferentes momentos históricos y sociales del desarrollo de la especie humana.

La sociedad contemporánea se encuentra inmersa (supeditada incluso) en una dinámica voraz de deconstrucción y reconstrucción de la discursividad que intenta definirla, en un esfuerzo de resignificación constante en la interpretación de los fenómenos sociales. Un común denominador pareciese ser la imperiosa necesidad de separarse de lo tradicionalmente utilizado, de los grandes discursos, de lo universal, de lo inamovible. La discursividad que impera sobre la dimensión cultural controla, margina o invisibiliza la corporalidad de todo aquello que es sujeto de control, ya sea el individuo o el yo colectivo referido por Foucault.

Los saberes, o más bien, el manejo y administración del conocimiento de la corporalidad, permiten el diseño e implementación de discursos y políticas que rigen las interacciones sociales. La administración del 
conocimiento ha ido adaptándose (camaleónicamente, vale decir) a cada época y realidad imperante. Desde la escritura cuneiforme hasta lo analógico-digital-virtual, influyendo dicho acondicionamiento tanto en forma como en fondo. Las múltiples expresiones (y su consecuente percepción) de lo no normativo en el espacio público, han sido tomadas por asalto bajo la etiqueta de lo políticamente correcto, donde queda de manifiesto una cautela extrema en todo cuanto se dice, cómo es dicho y por quiénes es dicho, ubicándose toda expresión bajo un riguroso escrutinio ético-moral.

En la actualidad, la reconfiguración discursiva pretende establecer criterios de conocimiento y apropiación en forma de discursos informativos direccionados en su carácter formativo hacia lo incluyente y el involucramiento de lo diverso, no como algo anormal, sino como una expresión más de la corporalidad. Los posicionamientos político-sociales LGBTTTIQ+ (lesbianas, gais, bisexuales, transgénero, travestidos, transexuales, intersexuales, queer y más) deben seguirse atentamente en cada una de sus etapas, analizar sus patrones y dinámicas al igual que las de aquellos que no se identifican o relacionan con esa discursividad incluyente, ya que las diferencias en sus planteamientos epistemológicos pudiesen posibilitar posicionamientos opuestos, polarizantes, enfocados en exaltar las diferencias en lugar de puntos de encuentro.

Desde una aproximación biopolítica, el involucramiento discursivo y operacional de la diversidad sexual y su corporalidad, además de multifactorial, es igualmente perceptible y cuantificable con base en tres ejes de análisis posibles, a saber: político, económico y sociocultural. Un ejemplo visible es la multiplicidad de miembros del colectivo LGBTTTIQ+, los cuales a través de su movilización y organización se han vuelto poseedores de personalidad y reconocimiento, no solo de tipo legal, sino económico y político; dicho estatus político-jurídico incrementa su visibilidad y espacios.

En el espacio del derecho, entendido este como un dispositivo que posibilita y gestiona las interacciones sociales en los ámbitos público y privado, Esposito indica que la interacción es posible solo a través de la distancia porque une a través de la separación, con relación a este punto señala que:

Aquí encontramos la última y más característica de las oposiciones asociadas con (o que dominan) la alternativa entre lo público y privado, las que en otras palabras contrastan communitas con immunitas. Si communis es aquel que es requerido para llevar a cabo las funciones de una oficina, o para la donación [elargizione] de una gracia, por el contrario, se llama inmune a aquel que no tiene que realizar oficina [inmunis dicitur qui nullo fungitur ofjicio], y por esa razón sigue siendo ingrato [ingratus] (Esposito, 2010, p. 6).

El individuo, como parte fundamental de la comunidad, adquiere no solo pertenencia sino sentido a partir de ella, su papel (y por tanto su valor desde una perspectiva pragmática) se encuentra supeditado a las funciones que debe cumplir y con esto aportar a su comunidad. Esposito señala que solo mediante el acto real de inmunización del individuo, este es liberado de su compromiso para-con-el-otro, sin necesidad de responder a un requerimiento colectivo, sino solo a cuestiones individuales.

La diversidad sexual (el colectivo LGBTTTIQ+) escapa a los parámetros tradicionalmente establecidos como funcionales en una sociedad, entre otros, su capacidad de formar biológicamente una familia. La función naturalmente requerida no puede ser satisfecha, por lo que dicho fin último de las políticas estatales proporcionó una justificación para la marginación; aquí no hay inmunización posible en el sentido "espositiano".

\section{Abriendo NUEVAS VÍAS de INVESTigación}

El conocimiento y análisis de los posicionamientos político-sociales LGBTTTIQ+, así como de su forma de organización, se vuelven imprescindibles para el entendimiento de las directrices y políticas que se gestionan y surgen en consecuencia de su activismo social, las cuales abarcan varias temáticas, desde necesidades básicas de salud, educación y seguridad, hasta aquellas propias de la vida política como la representatividad, actividades 
económicas, visibilidad social, reconocimiento de sociedades de convivencia, matrimonio, seguridad social y patrimonio, oportunidades de desarrollo laboral, representación y reconocimiento político. Las cuales se encuentran en proceso de transformación constante, superando andamiajes anquilosados y obsoletos de indiferencia, marginalidad y violencia.

Dentro de las prerrogativas derivadas de las movilizaciones, en el caso de las personas transexuales, destaca el derecho a solicitar libremente, a través de un riguroso y demandante acompañamiento médico-psicológico, los tratamientos de reacondicionamiento de tipo hormonal y cirugía de reasignación de sexo (CRS). Estos son procesos complejos, invasivos, prolongados y sensibles que impactan cada uno de los diferentes niveles de la autopercepción de identidad sexual y la vinculación social de las personas trans, en donde, además de las altas exigencias del procedimiento quirúrgico que modifican la forma sexual previa, el sostenimiento de la nueva forma y la calidad de vida posterior, también se generan pautas y retos propios.

La autopercepción y expresión de la diversidad sexual involucra complejos procesos de aceptación y reconocimiento, tanto en forma individual como colectiva. De manera similar estos procesos aplican dentro de los colectivos de la diversidad (en este caso el colectivo LGBTTTIQ+) y fuera de dicha diversidad en la sociedad en general. La diversidad sexual fue anteriormente catalogada clínicamente como una condición patológica, es decir, una enfermedad que se padecía. Sin embargo, a manera de reivindicación y con base en el involucramiento cada vez mayor de un lenguaje incluyente, dicho término ha sido modificado por el de expresiones no normativas o no binarias de la sexualidad, haciendo referencia a la pluralidad y matices de dichas expresiones, considerando categorías afectivas, emocionales y psicológicas. De esta manera, se han ido incorporando términos como género, orientación y preferencia. Es así que el elemento biológico (sexo) deja de ser el único criterio a considerar para la conformación de la sexualidad, pues otros aspectos como dinámicas, roles, factores socioculturales y ambientales son considerados igualmente significativos en dicho proceso.

Bennett señala que la aceptación de la identidad homosexual, en el caso específico de la homosexualidad masculina, entendida dicha identidad como aquellos elementos que definen y caracterizan un grupo en particular con sus gustos, preferencias, roles y dinámicas, está vinculada en numerosos casos con una cuestión de carácter innato, en contraste con otra de tipo electivo. En este sentido, Bennett (2014) menciona que: "El señalamiento de que uno nace lesbiana, gay, bisexual o transgénero se genera a manera de combatir las acusaciones de que el colectivo LGBTQ elige pertenecer a identidades no-normativas" (p. 214). La estructura de su planteamiento está basada en el argumento de que existe una programación original en vez de una que involucre una elección, lo que permite, al menos de manera discursiva, otorgarle un estatus de legitimidad, pues así la identidad homosexual adquiere una dimensión natural que la presenta como dada en lugar de elegida.

Por su parte, Sheldon, Pfeffer, Epstein, Feldbaum y Petty encontraron que aun cuando es diversa la respuesta y dependiente de cuestiones como la religión, formación académica, la edad y demás factores psico-sociales, hombres y mujeres anglosajones y afroamericanos consideraban la posibilidad del origen genético como un aspecto positivo que podría mejorar notablemente la percepción para con dicho colectivo LGBTTTIQ+. Al respecto señalan que: "La mayoría de las declaraciones (29 respuestas) de los participantes contenían la idea de que la información podía ser usada para educar a personas heterosexuales y ayudarles a ser más tolerantes" (Sheldon et al., 2007, p. 138), lo anterior posibilita la consideración del replanteamiento de lo innato sobre lo electivo.

Aun cuando el colectivo (LGBTTTIQ+) es una minoría en términos estadísticos, este posee una voluntad cohesionada, heterogénea discursivamente, pero con un fin común, lo cual posibilita una transición más fluida de lo discursivo a lo pragmático, con visibles resultados en los espacios tanto públicos como privados.

La discursividad del colectivo LGBTTTIQ+ no puede ser interpretada, incluso cuando metodológicamente pudiera resultar menos complejo analizarla de tal modo, como única y homogénea. No aplica sobre ella las mismas consideraciones y reglamentaciones para las especificidades de la homosexualidad 
en sus subcategorías: gais, lesbianas, bisexuales, transgénero, transexuales, travestidos, intersexuales, queer y más. Tampoco es viable homogenizarla respecto de su etnicidad racial, pues en el caso estadounidense comprenden anglosajones, afroamericanos, latinos, asiáticos, entre otros. En el caso mexicano con mestizos, pueblos originarios, afro-mexicanos, menonitas, etc. Como tampoco a través de su práctica religiosa que comprende católicos, protestantes, judíos, musulmanes, solo por nombrar algunas. La subcategorización o taxonomía del colectivo se vuelve no solo de interés semántico-discursivo, sino pragmático, pues las políticas públicas que busquen abarcarlas deberán considerarlas dentro de dicha planeación.

A manera de ejemplo, mientras que las visiones feministas pueden relacionar la opresión con el tradicional patriarcado, los gais y lesbianas pueden integrar otros elementos y a ellos sumar el prefijo hetero,volviéndose en heteropatriarcado. Por su parte, los bisexuales pudiesen considerar el género binario como un rasgo específico de dicha opresión y exclusión discursivas.

Obradors-Campos señala que se trata de un posicionamiento ontológico que tiene una amplia influencia sobre cómo son percibidos y comprendidos los géneros y sus relaciones dialécticas. El autor considera que: "Esta posición ontológica conlleva a aproximaciones epistemológicas basadas en una bipolar (muchas veces antagónica) y complementaria (muchas veces dependiente) concepción de tal dialéctica entre los géneros" (Obradors, 2011, p. 208).

Así propone un análisis minucioso de los términos y su uso al referir que la discriminación no es necesariamente sinónimo de opresión, pues mientras que la primera margina a través de la invisibilización, la segunda involucra mecanismos de control que constriñen, vulneran y violentan la libre expresión de la diversidad sexual. Invisibilidad y opresión operan alternada y simultáneamente la discursividad sobre la sexualidad y su diversidad; en ambos espectros, la vulnerabilidad del cuerpo diverso sigue siendo el objetivo primordial.

Como parte del análisis biopolítico, en cuanto a dispositivos estatales que administran la corporalidad, el sistema económico capitalista y sus pautas de consumo son, sin duda, un pilar del análisis de los dispositivos de control y manejo de la subjetividad de lo corporal. La visibilidad de los miembros del colectivo LGBTTTIQ + se ha visto beneficiada de tal dinámica y ha ido adquiriendo no solo una mayor proyección y alcances, sino que los estereotipos negativos otrora vinculados con la diversidad han ido experimentando modificaciones discursivas significativas.

La transición de dicha proyección involucra referentes positivos de dicha diversidad, sus miembros no son ya seres anormales, depravados o enfermos, a los que aludió el profundo análisis foucaultiano de los hospitales mentales de la Francia del siglo XIX, sino que son revalorizados; ahora son personas productivas y ampliamente involucradas con su comunidad y entorno, son generadores y consumidores de diferentes y variados productos y servicios especializados. Este proceso de resignificación responde, de igual manera, a una reconfiguración discursiva de las dinámicas de identidad y consumo que se benefician de la pluralidad de dichas expresiones de la sexualidad. Estas dinámicas afianzan su cohesión en distintos ámbitos y espacios a través de la generación de un arquetipo de identidad homosexual que se fortalece a medida que las políticas públicas se especializan en aspectos que arraigan dicha identidad.

La discursividad de la diversidad sexual con sus valores, preceptos y alcances, no escapa de los condicionamientos del biopoder como tampoco de los elementos biopolíticos que operan en sus márgenes y dentro de ella. Foucault puso especial atención en cómo la discursividad y el compromiso con lo dicho se vuelven un dispositivo de poder que condiciona las interacciones sociales, específicamente, las del Estadosistema y del cuerpo-individuo. En efecto, tal y como lo menciona Foucault (1977): "Saber cómo el individuo está vinculado y cómo acepta vincularse al poder que se ejerce sobre él es un problema jurídico, político, institucional e histórico" ( $\mathrm{p}$.28), siendo estos ejes jurídico-políticos e históricos sobre los cuales permea un poder de control institucional del individuo, en específico, de su corporalidad.

Un ejemplo de lo anterior es la confesión pastoral, pues esta se materializó como un mecanismo de compromiso de lo dicho y su correspondiente repercusión. La moral religiosa estableció un pacto con el 
Estado en donde ambos integraron un binomio que permitía una vigilancia estrecha de las actividades de lo privado, de lo que escapaba al escrutinio público, a diferencia de periodos como el del régimen feudal del medioevo en los que el secreto de todo acto impuro era el fin último. En contraste, bajo el esquema estatalreligioso francés de finales del siglo XIX, se fomentaba el hecho de externar dichas conductas con lujo de detalle, mientras más especificaciones mejor, radicando ahí el poder sobre el cuerpo falible.

Este referente de análisis puede ser aplicado directamente a la temática de la diversidad sexual y su posicionamiento político y sociocultural, pues la serie de normatividades y dispositivos pueden ser entendidos biopolíticamente en sus procesos e interacciones con las instituciones encargadas de administrar su movilidad, salud y representatividad, entre otros.

Llegados a este punto, cabe señalar que la base de la administración social ya no es la del conjunto, sino la del individuo colectivo y en este aspecto el Gobierno es un componente fundamental en tanto que, según Foucault (1977), desde el

Gobierno entendido en el sentido lato: manera de formar, transformar y dirigir la conducta de los individuos [...] se identifican tres grandes tipos de tecnologías: técnicas de producción de los objetos; técnicas de comunicación por las cuales los individuos se comunican entre sí, y técnicas de gobierno por las cuales los individuos actúan los unos sobre las conductas de los otros para alcanzar determinados fines u objetivos ( $\mathrm{p} .32$ ).

El poder, como menciona Foucault, debe ser ejercido, lo cual es posible debido a estructuras que le permiten administrar la corporalidad y su diversidad. El derecho, la normatividad, se vuelven instrumentos mediante los cuales la administración de los cuerpos es posible; a su vez, la delimitación y diferenciación de lo sano y lo enfermo, de lo moral y lo inmoral, son gestionados por dispositivos que regulan tanto al cuerpo social como al individual.

Agambem, por su parte, rastrea los orígenes de la orden o el mandato como punto de inflexión o de inicio del ejercicio del poder, ya que, no solo lo posibilita, sino que permea su desarrollo: "El origen nunca cesa de comenzar, es decir, nunca cesa de gobernar y mandar a lo que ha iniciado" (Agambem, 2012, p. 50). Todo aquello que manda, evoca o dispone, se encuentra jerárquicamente por encima de lo que de él se desprende, no solo por la acción que genera, sino por su carácter de ser primero. Las reglamentaciones o normatividades no solo permiten las relaciones e interacciones, sino que discursivamente las preceden y, por tanto, las condicionan y, en última instancia, las dominan. Lo que Agambem deja entrever es que dicho estatus se mantendrá aun cuando surjan nuevos reclamos sin importar cuan legítimos o radicales sean.

Por su parte, la propuesta foucaltiana, a través de su exhaustiva revisión de los procesos médico-legales (de la Francia del siglo XIX) y su consecuente andamiaje para el desarrollo de la biopolítica como disciplina, se centra en un análisis profundo (cuando más quirúrgico-discursivo) de las estructuras de poder, las cuales no interactúan con la sociedad (en el entendido de una mera operatividad) como un ente judicial, como tampoco con la corporalidad como singularidad, sino que la biopolítica representa más bien una ruptura del determinismo materialista clásico de las estructuras políticas a las que integra elementos biológicos, al considerarlos, epistemológicamente, componentes igualmente válidos, es decir, ubicados en el mismo nivel de relevancia que las estructuras mismas. Con referencia a esto, Lemke señala que Foucault parece tomar como punto de partida que:

La vida no denota la base ni el objeto de la política. En vez de eso, presenta una frontera para la política, una frontera que debe ser simultáneamente respetada y sobrepasada, una que parece ser ambos, natural y dada, pero también artificial y transformable (Lemke, 2011, pp. 4-5).

Lo biológico como frontera en cuanto límite debe ser superado; la biopolítica establece los parámetros que administran la vida a través de lo biológico, esto es, cómo debe darse el proceso de superación de dicha frontera, aun cuando a su vez establece otras nuevas, indefinidamente.

Tanto las instituciones como los individuos son urgidos, ya sea judicial o moralmente, a obedecer las disposiciones (aun cuando, según Agambem, no todas lo sean). La orden que manda, regula y coacciona, se 
fundamenta entonces en la dimensión de lo deseable y no tanto en lo existente; por lo tanto, se trata de una cuestión de voluntad, la cual se impone incluso al poder. En consonancia, Agambem continúa afirmando: "La hipótesis de Nietzsche era correcta: querer es ordenar. La voluntad es una orden y lo que es ordenado es la potencia. Entonces, el querer ordena al poder" (Agambem, 2012, p. 63).

\section{Cirugía de Reasignación de Sexo (CRS)}

La homosexualidad era considerada en Estados Unidos, hasta la década de los años 70, como un trastorno mental derivado de alteraciones genéticas y endocrinológicas; dicha consideración tenía influencia en el diseño e implementación de mecanismos para la administración y el manejo institucional de la diversidad sexual. En lo que respecta al tema Ardila argumenta lo siguiente:

Para clasificar las enfermedades mentales se utiliza generalmente el manual de la American Psychiatric Association titulado Diagnostic and Statistical Manual of Mental Disorders, DSM. En este manual, la homosexualidad se consideró una enfermedad mental hasta 1973 en que se removió de esa categoría [...] (Ardila, 2008, p.8).

Esta misma medida fue adoptada al año siguiente (1974) por la Asociación Americana de Psicología, lo cual representó un punto de inflexión y cambio de paradigma institucional respecto a la administración estatal de la diversidad sexual. Dicho cambio no surgió como un reconocimiento institucional de lo diverso como tal, sino que fue consecuencia de la amplia movilización y organización de la diversidad sexual que logró visibilizar y cuestionar los mecanismos institucionales que la habían administrado. A partir de entonces, el manejo biopolítico de la diversidad ha experimentado un proceso largo y complejo de transición, desde aquellos esquemas de persecución, marginalidad y victimización de la anormalidad a pautas más incluyentes y tolerantes.

En cuanto a la definición de transexual, en el DSM-5 (Diagnostic and Statistical Manual of Mental Disorders por sus siglas en inglés) se indica:

Denota a un individuo que busca, o que ha sufrido, una transición social de hombre a mujer o mujer a hombre, que en muchos casos, pero no en todos, también implica una transición somática por tratamiento con hormonas cruzadas y cirugía genital (cirugía de reasignación de sexo) (American Psyquiatric Association, 2013, p. 451).

Por su parte, la Clasificación Internacional de Enfermedades (CIE) en su décima primera versión publicada en línea en el año 2019, titulada Estadisticas de morbilidad y mortalidad, en su apartado de "Discordancia de género" y, específicamente, en lo concerniente a la adolescencia y la adultez, refiere que:

La discordancia de género en la adolescencia o adultez se caracteriza por una discordancia marcada y persistente entre el
género experimentado por la persona y el sexo asignado, lo que a menudo conduce a un deseo de "transición" para vivir
y ser aceptada como una persona del género experimentado, ya sea por medio de un tratamiento hormonal, intervención
quirúrgica u otros servicios de salud, para que el cuerpo pueda alinearse, tanto como lo desee y en la medida de lo posible, con el
género experimentado. El diagnóstico no se puede hacer antes del inicio de la pubertad. Las variaciones en el comportamiento
de género y las preferencias no constituyen por sí solas una base para determinar el diagnóstico" (OMS- CIE, 2019, HA60,
párr. 1).

La exposición a hormonas masculinizantes (testosterona), tanto en periodos prenatales (antes del nacimiento) como posnatales (después del nacimiento), pueden influir en la formación y maduración del sexo biológico (cariotípico) y sus respectivos órganos, a diferencia de la configuración promedialmente mayoritaria heterosexual, en la que se presenta cierta regularidad en la exposición a algunas hormonas como es el caso de la testosterona. Los rangos de exposición (aumentada o disminuida) a la hormona testosterona en periodos prenatales o posnatales en los cariotipos tanto XY como XX pueden (no de forma totalmente esclarecida aún) dar origen a valores y "especificidades" propias que se alejan de los rangos promediales, los cuales a su vez pudiesen presentar pautas de orientación y preferencia sexual no normativas. 
Al respecto Castellanos, Bao y Swaab (2017) mencionan que: "El fenotipo del embrión humano es bipotencial. En individuos XY, la diferenciación sexual comienza prenatalmente con los efectos virilizantes de la testosterona, y por este efecto virilizante, obtenemos la diferencia sexual” (p. 280). La diferenciación sexual (dimorfismo), según estos autores, está mediada por la hormona testosterona en cuanto al desarrollo y especialización de ciertas estructuras, entre ellas, la red testicular en los varones y, en ausencia de esta hormona, conduciría a la formación de órganos sexuales femeninos primarios y secundarios.

La relación del sexo biológico con la identidad sexual presenta retos importantes para su análisis. La convivencia entre la corporalidad física y la autopercepción sexual de la persona, se encuentra sujeta a diversos factores, no solo genéticos (genotípicos) sino psico-sociales que derivan en dinámicas de pertenencia, asociación, identidad, entre otros.

En el caso particular de la transexualidad, Becerra (2003) menciona que: "La transexualidad es un trastorno de identidad de género (TIG) por el cual una persona de un sexo biológico determinado se siente pertenecer al contrario" ( p .23). Bajo dicha consideración, la transexualidad era aún considerada, desde el ámbito médico, como un trastorno en el que no existe una concordancia entre el sexo biológico y el género auto percibido de la persona que lo manifiesta.

El DSM-5, en su versión más actualizada, ha modificado el término trastorno de identidad de género, el cual utilizaba en su versión anterior (DSM-4), por el de disforia de género. En esta última versión se señala que: "El término actual es más descriptivo que el término anterior DSM-4 trastorno de identidad de género y se enfoca en la disforia como el problema clínico, no en la identidad per se" (Asociación Americana de Psiquiatría, 2013, p. 451).

Por su parte, Hembree et al. rastrea los primeros usos médico-clínicos del término transexual. Citando a Meyerowitz estos autores señalan que: “[...] fue Hirschfeld quien acuñó el término "transexual” en 1923 para describir a personas que desean vivir una vida que corresponda con su género percibido en contraste con su género designado" (Hembree et al., 2017, p. 3873). Respecto a la actualización del término transexual y con base en el DSM-5, los autores mencionan que se utiliza el término género-disfórico/ género-incongruente para referirse a aquellas personas que experimentan incongruencia entre su modo de vivir y su género biológico.

De igual forma, Hembree et al. alude a que el término trastorno de disforía de género fue modificado por el de disforía de género en la CIE-11 al igual que en el DSM-5. Haciendo, además, el señalamiento puntual de que los conocimientos de las interacciones biológico-ambientales se encuentran aún en estudio y sujetos a un proceso de actualización constante, se hace referencia que:

En resumen, aunque todavía se desconoce mucho sobre la identidad de género y su expresión, los estudios convincentes respaldan el concepto de que los factores biológicos, además de los factores ambientales, contribuyen a este aspecto fundamental del desarrollo humano (Hembree et al., 2017, p. 3875).

Incluso, cuando el término ha cambiado por uno menos estigmatizante, la disforia de género (incongruencia entre el sexo biológico y el género), en su descripción aún es visto como un trastorno de personalidad, el cual debe ser atendido a través de tratamientos integrales (psico-farmacológicos).

Según el DSM-5, tal condición, o más bien, tal especificidad suele mantener cierta correlación estadística entre adolescentes y su tránsito a la adultez. En este sentido, se menciona que:

Las expresiones de disforia anatómica se vuelven más comunes a medida que los niños con disforia de género se acercan y anticipan a la pubertad. Las tasas de persistencia de la disforia de género desde la infancia hasta la adolescencia o la edad adulta varían. En los varones natales, la persistencia ha oscilado entre el 2,2\% y el 30\%. En las mujeres natales, la persistencia ha oscilado entre el 12\% y el 50\% (Asociación Americana de Psiquiatría, 2013, p. 451).

Escapa a la intención de este análisis profundizar sobre las múltiples interpretaciones en torno al binomio sexo-género y su performatividad, por lo que solo centra su atención en la transexualidad como referente 
epistemológico que posibilita manejar las similitudes, diferencias e interacciones entre sexo biológico e identidad sexual.

Para el abordaje de la transexualidad (género-disfórico/género-incongruente) como fenómeno psicobiológico y social complejo, es pertinente una descripción del procedimiento fármaco-quirúrgico de la Cirugía de Reasignación de Sexo (CRS), considerada hasta el momento como el más invasivo y definitivo procedimiento que busca hacer corresponder las dimensiones de la autopercepción corporal de sexo y género a través de la modificación de características sexuales tanto en pacientes masculino-femenino (M-F) como femenino-masculino (F-M), entre las cuales se encuentran modificaciones en cuanto a vello facial y corporal, tono de voz (más agudo o grave), implantación de mamas (o remoción de estas) hasta la reconfiguración de los órganos sexuales, vaginoplastía y prótesis peneana en el caso de las F-M y penectomía en el de las M-F.

El sentir y actuar de quienes se someten a un procedimiento de tal magnitud suele no ser comprendido a profundidad y, por ende, suele no ser bien recibido por parte del círculo social inmediato de quienes lo experimentan; esto derivado, en principio, por el desconocimiento de lo complejo y delicado que dicho proceso representa sumado, además, a que distintos sectores de la sociedad desconocen las particularidades y los retos que representa, a lo cual se adiciona la carga ético-moral tradicionalista imperante.

En España, la reasignación de sexo cuenta con mecanismos médico-legales que han ido permitiendo que las instituciones estatales tomen y fijen posturas y acciones respecto de dicho fenómeno. En Madrid, la Unidad de Trastornos de Género del Hospital Ramón y Cajal ha representado una opción para el abordaje, tratamiento y seguimiento de la transexualidad (género-disfórico/género-incongruente). El nombre de la unidad hospitalaria adoptó inicialmente el término trastorno como parte de las siglas en su nombre; sin embargo, en el 2016 y derivado de las modificaciones promovidas por la legislatura de la comunidad de Madrid, en congruencia con el DSM-5, cambió su nombre por el de Unidad de Identidad de Género, siendo hasta el 2018 cuando se dio la remoción del término transexual del DSM-5 por parte de la Organización Mundial de la Salud (OMS).

En dicha unidad del hospital, se implementa un abordaje interdisciplinario médico-psicológico que brinda acompañamiento al paciente durante su complejo proceso de reasignación de sexo. Respecto de la identidad sexual, Asenjo, Rodríguez, Lucio y Becerra (2011) mencionan que: "La identidad sexual es el factor subjetivo íntimo que establece la convicción interior de pertenecer a un sexo determinado” (p. 88).

Cuando por causas genéticas, socioculturales o una combinación de ambas se presenta una desarmonización entre la relación sexo biológico y género, la unidad brinda apoyo y acompañamiento para que el paciente pueda cambiar de sexo, pero dicho proceso cuenta con rigurosos protocolos que deben cumplirse para su desarrollo. Este procedimiento comprende varias fases que preparan el reacondicionamiento, las cuales van desde el abordaje interdisciplinar inicial (médico-psicológico), el tratamiento hormonal y la experiencia de vida real, la cirugía para el cambio de caracteres secundarios, además de la propia CRS que comprende también el seguimiento del paciente pos-CRS.

El procedimiento de reasignación de sexo comprende diferentes estadios que dan inicio con el reconocimiento y la aceptación de la propia homosexualidad por parte del paciente, la valoración clínica o legitimidad de la autopercepción genuina de una des-armonización entre su sexo biológico y su género, esto quiere decir que no sea consecuencia de derivación secundaria de algún proceso traumático o compromiso neurológico-endocrinológico, pues ante alguno de dichos escenarios el manejo y la canalización toma un curso distinto.

La identidad de género del paciente es valorada a través de una serie de minuciosas entrevistas interdisciplinarias médico-psicológicas (cuestionarios y valorización clínica, estudios de imagenología y endocrinológicos) por parte del personal médico calificado en dicha temática. Además, se presenta el ajuste de los roles y las dinámicas sociales respecto de su nueva identidad en su círculo social y laboral. Dicha transición incluye asumir por parte del paciente sus nuevas coordenadas y desplazarse en ese nuevo registro, el cual debe ser supervisado a través de un cercano seguimiento médico-psicológico. 
De esta forma, se incluyen paulatinamente los tratamientos de nivelación hormonales, masculinizantes o feminizantes, según sea el caso, dichos tratamientos se valoran de manera constante. Posteriormente, y solo a través de un diagnóstico favorable por parte del equipo interdisciplinario, el paciente clasifica como candidato para someterse a la CRS, la cual no es la culminación del proceso en sí, sino un paso más de este, pues el tratamiento hormonal y psicológico debe mantenerse durante el resto de la vida de la persona, además, de mantener una estrecha vigilancia y atención multidisciplinaria de posibles complicaciones derivadas del procedimiento quirúrgico realizado y de posibles repercusiones relacionadas con la edad derivadas de esta.

\section{Conclusiones}

La diversidad sexual tiene en estos tiempos dos vertientes discursivas mayoritarias y espectralmente polarizantes (sin que esto limite las múltiples expresiones que transitan entre ambas). Por un lado, el discurso incluyente con aliados tanto dentro como fuera del colectivo que potencializan, con base en la proyección, el apoyo y la defensa de una identidad colectiva, la cual lejos de debilitarse encuentra cada vez más seguidores y adeptos. Se le suma además la promoción de una nueva configuración socio-institucional para su involucramiento, desarrollo y consolidación.

Mientras que, en contraste, existe una creciente animadversión radicalizada por sectores neoconservadores que no solo cuestionan la validez de dicha identidad colectiva, sino que ponen en entredicho las bases ideológicas que la sustentan y las modificaciones institucionales que de estas surgen, siendo el discurso éticomoral tradicional y religioso el que configura directa o indirectamente la mayoría de estas discursividades.

La complejidad biológica primigenia, aunada a la psicosocial, pudiese verse beneficiada con un análisis transdisciplinario que permite interpretar diferentes elementos propios de tan intrincado fenómeno. La filosofía, por ejemplo, puede aportar perspectivas ontológicas y axiológicas para una taxonomía ético-moral de la corporalidad humana y su sexualidad, a través de una visión de conjunto que vincule la experiencia sensible personal y su interrelación con el entorno inmediato. La biopolítica, por su parte, puede aportar elementos de juicio que permitan dimensionar y valorar la importancia de los factores biológicos en la administración (diseño, implementación y seguimiento) de las políticas públicas que administran al individuo y su corporalidad.

La relación del binomio sexo-género ha sido motivo de diversas interpretaciones, ideologías y consideraciones en diferentes épocas y latitudes. Más allá de lo puramente discursivo (histórico-social), el retorno a la revisión del componente biológico inmunizado de intolerancias y encasillamientos permite identificar elementos que escapan a una discursividad enfocada únicamente en aquellos componentes de carácter identitario y psico-emocional.

La consideración tradicional del sexo biológico respecto de la información cromosómica XX o XY y sus niveles intermedios abordaba únicamente el sexo cigótico o cromosómico; sin embargo, otras categorías no suelen ser consideradas en el análisis discursivo, siendo excluidas otro tipo de variantes como la génica, cromatínica, cariotípica, gonadal, ductal, genital externa, de caracteres secundarios, asignación, crianza y psicológica, categorías que son igual y epistemológicamente relevantes en el análisis y comprensión de la conformación integral psico-biológico y social del sexo.

Este abordaje ha permitido identificar el caso concreto de la atención institucional de las personas transexuales en tan intrincado trance de reacondicionamiento sexual. El Estado, mediante la implementación de dichos mecanismos médico-legales ha logrado, aunque de forma limitada, hacer frente a un fenómeno social que va en aumento y que, por ende, reclama un manejo interinstitucional cada vez más integral, el cual requiere de condiciones específicas como infraestructura, presupuestos y legislación para su operatividad. Dichos elementos deben ser analizados con detenimiento, con el fin de evitar que sesgos ideológicos o intereses sectoriales afecten su funcionamiento. 
En cuanto al enfoque biopolítico, la administración de los cuerpos por parte del Estado español busca adecuarse a las múltiples expresiones y reclamos de quienes le conforman. La organización social de las minorías (como la sexual) ha ido conquistando espacios y reivindicaciones que han permitido, de manera inicial, no solo combatir la marginalización, la violencia o la invisibilidad, sino también reconfigurar socialmente las instituciones para que estas respondan a la serie de expectativas que la pluralidad requiere y que, paulatinamente, va integrando en sus legislaciones asegurando su cumplimiento.

Dentro de las consideraciones que apenas comienzan a formar parte de la discusión biopolítica y su puesta en práctica dentro de la administración de la salud en México, respecto de la transexualidad y la salud pública, todavía no se contemplan los marcos legales que establezcan los protocolos institucionales para la atención médica de las personas transexuales que desean someterse a un procedimiento quirúrgico de reasignación de sexo. Aunque existen protocolos para la atención médica que se apegan a las disposiciones de los Derechos Humanos, tanto nacionales como internacionales, en cuanto al trato digno de los pacientes, el manejo adecuado de su información personal y demás parámetros, aún no existe una institución que canalice y de seguimiento a las condiciones de dichos pacientes; por su parte, la Ciudad de México se encuentra en vías de establecer las primeras atenciones de este tipo en el país.

Es importante mencionar que, además del proceso de reasignación de sexo como tal, debe existir un adecuado monitoreo y seguimiento de posibles complicaciones posquirúrgicas de los pacientes que se han sometido ha dicho procedimiento, entre las cuales pueden presentarse: estenosis uretrales, cuadros de litiasis vesicales, hernias inguinales, fístulas urinarias y obstrucción de conductos espermáticos, por nombrar algunas. Estas condiciones afectan aún más un proceso de por sí complejo y demandante, donde la calidad y expectativa de vida de los pacientes que se someten a este tipo de cirugías se ven constantemente vulneradas. Sumado a esto figuran las fallas, deficiencias u omisiones en el proceso de reacondicionamiento de las dinámicas sociales de la persona transexual con sus círculos familiares, sociales y laborales inmediatos, de ahí la importancia del abordaje multidisciplinario de dicho fenómeno.

Consideramos válida una aproximación transdisciplinaria de la discursividad de la reconfiguración sexogénero, como un recurso metodológico con aplicación a diferentes elementos que componen el fenómeno de la diversidad sexual y su interacción con las múltiples dinámicas que administran la expresión sexual de la corporalidad.

Entendidas dichas expresiones como la autopercepción, presencia e interacciones entre la diversidad sexual (colectivo LGBTTTIQ+) y la sociedad en general, los hallazgos en materia de la ingeniería genómica son un referente que permite identificar las especificidades de los cuerpos en transición a sus nuevas coordenadas corporales y, a su vez, ayudar a valorar cómo estas pueden o no influir en patrones de comportamiento, manejo institucional y aceptación social.

El enfoque transdisciplinario sugerido, que involucra tanto factores biológicos como socio-culturales, pretende proveer pautas para un abordaje integral de lo complejo y multifactorial de dicho proceso de resignificación. Es preciso señalar que las controversias propias del intercambio entre posturas a favor y en contra de la diversidad sexual, negadas al diálogo recíproco, mantienen dinámicas que imposibilitan toda construcción de puentes que vinculen lo alternativo con lo promedial. La reincorporación del elemento biológico permite considerar perspectivas que amplían el conocimiento de las especificidades, lo cual ayuda a disolver discursos de odio basados en el desconocimiento de la diversidad sexual.

El manejo y confrontación de las situaciones reales con las bases informativas científicas quizá pueda ayudar a construir nuevos planteamientos biopolíticos, desde una filosofía del cuerpo, que permitan una visión ampliada de la diversidad sexual, no en busca de una vinculación simulada y superficial de lo alternativo, sino de la inclusión basada en el conocimiento y reconocimiento de ese otro, integrando así lo diverso y sumando lo similar. En este sentido, es relevante profundizar en las nociones del cuerpo sexuado que se encuentran en juego detrás de cada postura de género, pues se vuelven no solo una herramienta teorizante que permite diseccionar minuciosamente los componentes, condicionantes y situaciones de la evolución social 
del fenómeno en cuestión, sino que también pueden ofrecer referentes clave para, desde allí, ejercer su examen crítico y generar posibles alternativas de aproximación a tan complejo fenómeno.

\section{ReFERENCIAS}

Agambem, G. (2012). Teología y lenguaje. Del poder de Dios al juego de los niños. Buenos Aires: Las Cuarenta.

Ardila, R. (2008). Homosexualidad y psicología. Bogotá: El Manual Moderno.

Asenjo, N., Rodríguez, J.M., Lucio, M.J. y Becerra, A. (2011). Abordaje multidisciplinar de la transexualidad: desde atención primaria a la Unidad de Trastornos de Identidad de Género de Madrid (UTIG MADRID). Revista Española de Medicina de Familia- Semergen, 37(2), 87-92.

Asociación Americana de Psiquiatría. (2013). Sección II. Criterios y códigos de diagnóstico. En Manual diagnóstico y estadístico de los trastornos mentales (5. ${ }^{\mathrm{a}}$ ed.). Recuperado de http://dx.doi.org/10.1176/appi.books. 9780890 425596

Becerra, A. (2003). Transexualidad: la búsqueda de una identidad. Madrid: Díaz de Santos.

Bennett, J. (2014). Born This Way: Queer Vernacular and the Politics of Origins. Communication and Critical/ Cultural Studies, 11(3), 211-230.

Castellanos, L., Bao, A. \& Swaab, D. (2017). Sexual Identity and Sexual Orientation. En D.W. Pfaff y M. Joels (Eds.), Hormones, Brain and Behaviour (pp. 279-290). United States: Academic Press.

Esposito, R. (2010). Communitas. The Origin and Destiny of Community. California: Stanford University Press.

Foucault, M. (1977). Obrar mal y decir la verdad. México: Editorial Siglo XXI.

Foucault, M. (1979). Microfisica del poder. Madrid: Las Ediciones de la Piqueta.

Hembree, W., Cohen, P., Gooren, L., Hannema, S., Meyer, W., Murad, M.,...T’ Sjoen, G. (2017). Endocrine Treatment of Gender-Dysphoric/Gender-Incongruent Persons: An Endocrine Society Clinical Practice Guideline. The Journal of Clinical Endocrinology \& Metabolism, 102(11), 3869-3903.

Le Breton, D. (2002). Antropología del cuerpo y modernidad. Buenos Aires: Ediciones Nueva Visión.

Lemke, T. (2011). Biopolitics: An Advanced Introduction. Nueva York: New York University Press.

Obradors, M. (2011). Deconstructing Biphobia. Journal of Bisexuality, 11(2-3), 207-226.

Organización Mundial de la Salud. (2019). Clasificación internacional de enfermedades (11. $\left.{ }^{\mathrm{a}} \mathrm{ed}.\right)$. Recuperado de htt ps://icd.who.int/browse11/1-m/es

Rico Bovio, A. (2017). Muerte y resurrección del cuerpo. México: Plaza y Valdés.

Sheldon, J., Pfeffer, C., Epstein, T., Feldbaum, M. \& Petty, E. (2007). Beliefs About the Etiology of Homosexuality and About the Ramifications of Discovering Its Possible Genetic Origin. Journal of Homosexuality, 52, 111-150.

\section{Notas}

1 Según el diccionario Cambridge, el adjetivo transexual se utiliza para describir a alguien que siente que no es del mismo género (género entendido aquí como sexo) que el cuerpo físico con el que nació o que no encaja fácilmente en ser hombre o mujer.

2 La cirugía de reasignación de sexo comprende un procedimiento endocrinológico y quirúrgico que permite modificar la apariencia de caracteres sexuales secundarios, como lo es el caso de los genitales externos, mamas, vello corporal, tono de voz, entre otros. 